\title{
LOW-COMPLEXITY HIGH-PERFORMANCE GFSK RECEIVER WITH CARRIER FREQUENCY OFFSET CORRECTION
}

\author{
Charles Tibenderana and Stephan Weiss \\ Communications Research Group \\ School of Electronics \& Computer Science \\ University of Southampton, UK
}

\begin{abstract}
This paper presents an implementation of a GFSK receiver based on matched filtering of a sequence of $K$ successive bits. This enables improved detection and superior BER performance but requires $2^{K}$ matched filters of considerable complexity. Exploiting redundancy by performing phase propagation of successive singlebit stages, we propose an efficient receiver implementation. Results presented highlight the benefits of the proposed method in terms of computational cost and performance compared to standard methods. We also address carrier frequency offset, and suggest a blind algorithm for its elimination. Performance results are exemplarily shown for a Bluetooth system.
\end{abstract}

\section{INTRODUCTION}

Gaussian Frequency Shift Keying (GFSK) is a bandwidth preserving digital modulation technique, which has been used for low-cost transmission standards such as Bluetooth. This low cost makes it an attractive alternative over expensive high data rate WLAN services such as IEEE802.11b. Therefore, in this contribution we aim at deriving GFSK receivers for high performance data transfer, which can enable their use in inexpensive standards similar to Bluetooth more efficiently.

High performing receivers for GFSK use a system of filters to match expected waveforms [1], or the Viterbi algorithm to penalise illegitimate phase transitions [2]. Basic reception methods include FM-AM conversion, phase-shift discrimination, zero-crossing detection, and frequency feedback [3]. Susceptibility of GFSK to carrier frequency errors necessitates additional functions to handle carrier offset conditions. Current research into frequency correction has focused on adaptive thresholding based on the DC level of a training signal [4], while researchers on blind methods have considered use of frequency difference detectors [5], and excess mean-square algorithms, whereby the error function directs the loop towards the point of equilibrium $[6,7]$.

We adopt a high-performance GFSK receiver that achieves near optimum performance in AWGN [1] but uses a prohibitively complex bank of filters to match a large set of legitimate waveforms over several bit intervals. However, we reduce the computational cost by performing filtering over a single bit interval, and propagating the results over successive bit periods, thereby eliminating redundancy in providing the matched filter outputs. We also propose a blind algorithm for carrier frequency correction. It is based on the observation of the phase gain in the transmit signal over a finite time-interval. Our derivation concurs with work by other researchers [7].
Hence, this paper will, based on a brief review of the standard high-performance receiver in Sec. 3, introduce a novel lowcomplexity high-performance algorithm in Sec. 4. A simple recursive adaptive algorithm for carrier offset correction is derived in Sec. 5. Sec. 6 discusses the results before we conclude in Sec. 7.

\section{SIGNAL MODEL}

GFSK generally modulates a multilevel symbol $p[k]$, which here is assumed to be binary, $p[k] \in\{ \pm 1\}$. This bit sequence is expanded by a factor of $N$ and passed through a Gaussian filter with impulse response $g[n]$ of length $L N$, thus having a support of $L$ bit periods and yielding a continuous instantaneous angular frequency signal

$$
\omega[n]=2 \pi h \sum_{k=-\infty}^{\infty} p[k] g[n-k N],
$$

where $h$ is the modulation index. The phase of the baseband version of the transmitted signal,

$$
s[n]=\exp \left\{j \sum_{\nu=-\infty}^{n} \omega[\nu]\right\}=\prod_{\nu=-\infty}^{n} e^{j \omega[\nu]},
$$

is determined as the cumulative sum over all previous phase values $\omega[n]$.

We assume that the received signal, $r[n]$, has been subject to a gain $A$ and distortion by additive white Gaussian noise (AWGN) $v[n]$ that is uncorrelated with the transmitted signal $s[n]$,

$$
r[n]=A s[n] e^{j \Delta \Omega n}+v[n]
$$

whereby clock tolerances cause a carrier frequency offset $\Delta \Omega$ relative to the transmitter.

\section{MATCHED FILTER BANK RECEIVER}

A standard high-performance receiver is discussed in $[1,8]$, which achieves near-optimum non-coherent estimation of a bit in AWGN. This method is based on a filter bank containing all possible transmitted sequences $s[n]$ over a duration of $K$ bit periods. Over this observation interval, due to the support length of the Gaussian filter, $2^{K+L-1}$ possible sequences exist apart from an initial phase shift. The best matching filter then determines the detected value of the middle bit in the $K$ bit sequence, assuming $K$ to be odd. In order to reduce the large complexity of this receiver, the marginal bits influencing the $K$ bit sequence are often omitted [1]. The resulting scheme assumes $s_{i, j}[n]$ different possible transmitted sequences with $i \in\{ \pm 1\}$ indicating the value of the middle bit and 
$j=0(1) 2^{K-1}$ indexing the possible combinations of the remaining $K-1$ bits. To determine the output bit $\hat{p}[k]$ of the receiver, a detector selects the matched filter output with the largest magnitude according to

$$
\hat{p}[k]=\arg \max _{i}\left|\sum_{n=0}^{K N-1} r[k N-n] \cdot s_{i, j}^{*}[-n]\right|,
$$

where $s_{i, j}^{*}[-n]$ are the $2^{K}$ matched filter responses. The performance of this receiver improves when increasing the observation interval $K$. However, despite its performance merits and neglecting $L-1$ marginal bits, the computational complexity of

$$
C_{\text {standard }}=4 N K 2^{K}=N K 2^{K+2}
$$

real valued multiply accumulates (MACs) is prohibitive. Therefore, in the following we seek a low complexity implementation of this receiver.

\section{LOW-COMPLEXITY RECEIVER}

We will first inspect the matched filter responses in Sec. 4.1, and thereafter develop a recursive scheme for their representation in Sec. 4.2, leading to an analysis of its complexity in Sec. 4.3.

\subsection{Received Signals}

Let us assume that $K$ bit periods of the received signal $r[n]$, for simplicity here without carrier offset, are held in a tap delay line (TDL) vector $\mathbf{r}_{k}$, synchronised with the $k$ th bit to be the most recent datum:

$$
\mathbf{r}_{k}=\left[\begin{array}{c}
\tilde{\mathbf{r}}_{k} \\
\tilde{\mathbf{r}}_{k-1} \\
\vdots \\
\tilde{\mathbf{r}}_{k-K+1}
\end{array}\right]=A \mathbf{s}_{k}+\mathbf{v}_{k}=A \cdot \underbrace{\left[\begin{array}{c}
\tilde{\mathbf{s}}_{k} \\
\tilde{\mathbf{s}}_{k-1} \\
\vdots \\
\tilde{\mathbf{s}}_{k-K+1}
\end{array}\right]}_{\mathbf{s}_{k}}+\mathbf{v}_{k}
$$

where $\mathbf{v}_{k} \in \mathbb{C}^{N K}$ holds the noise samples. The vectors $\tilde{\mathbf{s}}_{k}$, and analogously $\tilde{\mathbf{r}}_{k}$, are defined as

$$
\tilde{\mathbf{s}}_{k}=\left[\begin{array}{lllll}
s[k N] & s[k N-1] & \ldots & s[(k-1) N+1
\end{array}\right]^{\mathrm{T}}
$$

According to (2), $\tilde{\mathbf{s}}_{k}$, holding $N$ samples within a bit period, can be expanded as

$$
\tilde{\mathbf{s}}_{k}=\underbrace{\left[\begin{array}{c}
\prod_{\nu=(k-1) N+1}^{k N} e^{j \omega[\nu]} \\
\prod_{\nu=(k-1) N+1}^{k N} e^{j \omega[\nu]} \\
\vdots \\
e^{j \omega[(k-1) N+1]}
\end{array}\right]}_{\mathbf{u}_{k}} \cdot \prod_{\nu=-\infty}^{(k-1) N} e^{j \omega[\nu]}
$$

whereby for the samples in $\mathbf{u}_{k}$ the instantaneous frequency is only accumulated from the start of the $k$ th bit period. Inserting (8) into $\mathbf{s}_{k}$ yields

$$
\mathbf{s}_{k}=\left[\begin{array}{cl}
\mathbf{u}_{k} & \cdot e^{j\left(\theta_{k-K+1}+\cdots+\theta_{k-2}+\theta_{k-1}\right)} \\
\mathbf{u}_{k-1} & \cdot e^{j\left(\theta_{k-K+1}+\cdots+\theta_{k-2}\right)} \\
\vdots & \\
\mathbf{u}_{k-K+2} & \cdot e^{j \theta_{k-K+1}} \\
\mathbf{u}_{k-K+1} & \cdot 1
\end{array}\right] \cdot e^{j \alpha},
$$

with

$$
\theta_{k}=\sum_{\nu=(k-1) N+1}^{k N} w[\nu] \quad \text { and } \quad \alpha=\sum_{\nu=-\infty}^{(k-K) N} w[\nu] \text {. }
$$

Firstly, note that each vector $\mathbf{u}_{m}$ can take on the shape of $2^{L}$ different waveforms, whereby $L$ was the support length of the Gaussian window in bit periods. Secondly, observe that a phase correction term $e^{j \theta_{k}}$ contains the instantaneous frequency values accumulated over the $k$ th bit period, which is held in the top element of $\mathbf{u}_{k}$ in (8) and is applied to all subsequent bit periods. The initial phase of $s[n]$ entering the TDL is $\alpha$.

\subsection{Recursive Matched Filter Formulation}

The matched filter responses $s_{i, j}^{*}[-n]$ are designed from the transmitted signal $s[n]$ in (2). Utilising the previous observation that $\mathbf{u}_{k}$ only takes on $2^{L}$ basic waveforms independent of $k$, we will construct a matched receiver in steps.

Case $K=1$. Consider a matched filter for $K=1$ covering the $k$ th bit period. The $2^{L}$ matched filter outputs are given by

$$
\mathbf{y}_{k}^{(1)}=\mathbf{W}^{(1)} \tilde{\mathbf{r}}_{k}
$$

with $\mathbf{W}^{(1)} \in \mathbb{C}^{2^{L} \times N}$ containing the possible complex conjugated waveforms in its rows. The superscript ${ }^{(1)}$ indicates that only a single bit period $K=1$ is observed. The first column of $\mathbf{W}^{(1)}$, denoted by $\mathbf{w}$, holds the $2^{L}$ possible values for $e^{j \theta_{k}}$.

We assume that the first row of $\mathbf{W}^{(1)}$ is the matched filter for $L$ bits of value -1 , binary coded decimally down to the last row with $L$ bits of value +1 .

Case $K=2$. Expanding to $K=2$, we can denote

$$
\mathbf{y}_{m}^{(2)}=\mathbf{W}^{(2)}\left[\begin{array}{c}
\tilde{\mathbf{r}}_{k} \\
\tilde{\mathbf{r}}_{k-1}
\end{array}\right] .
$$

In constructing the $2^{L+1}$ matched filter responses in $\mathbf{W}^{(2)}$, only one extra bit needs to be considered compared to the responses in $\mathbf{W}^{(1)}$. Thus each sequence in $\mathbf{W}^{(1)}$ can be doubled up and expanded by an extra bit input, enabling to write

$$
\begin{aligned}
\mathbf{y}_{k}^{(2)} & =\mathbf{D}^{(2)} \mathbf{A}^{(2)} \mathbf{W}^{(1)} \tilde{\mathbf{r}}_{k}+\mathbf{M}^{(2)} \mathbf{W}^{(1)} \tilde{\mathbf{r}}_{k-1} \\
& =\mathbf{D}^{(2)} \mathbf{A}^{(2)} \mathbf{y}_{k}^{(1)}+\mathbf{M}^{(2)} \mathbf{y}_{k-1}^{(1)}
\end{aligned}
$$

whereby $\mathbf{y}_{k-1}^{(1)}$ are the single bit matched filter outputs for the $(k-1)$ st bit. The matrix $\mathbf{A}^{(2)}$,

$$
\mathbf{A}^{(2)}=\text { blockdiag }\left\{\left[\begin{array}{l}
1 \\
1
\end{array}\right]\right\} \in \mathbb{Z}^{2^{L+1} \times 2^{L}},
$$

produces an extra copy of each response in $\mathbf{W}^{(1)}$, while

$$
\mathbf{D}^{(2)}=\left[\begin{array}{cc}
\operatorname{diag}\{\mathbf{w}\} & \mathbf{0} \\
\mathbf{0} & \operatorname{diag}\{\mathbf{w}\}
\end{array}\right] \in \mathbb{C}^{2^{L+1} \times 2^{L+1}}
$$

applies the phase correction term $e^{j \theta_{k}}$, and the matrix

$$
\mathbf{M}^{(2)}=\left[\begin{array}{l}
\mathbf{I}_{2^{L}} \\
\mathbf{I}_{2} L
\end{array}\right] \in \mathbb{Z}^{2^{L+1} \times 2^{L}}
$$


is assigning the expansion by the extra bit consider for $K=2$, whereby $\mathbf{I}_{2^{L}}$ is an $2^{L} \times 2^{L}$ identity matrix.

Case $K$ arbitrary. Generalising from the previous cases, we formulate recursively for $\mathbf{y}_{k}^{(K)} \in \mathbb{C}^{2^{K+L-1}}$

$$
\mathbf{y}_{k}^{(K)}=\mathbf{D}^{(K)} \mathbf{A}^{(K)} \mathbf{y}_{k}^{(K-1)}+\mathbf{M}^{(K)} \mathbf{y}_{k-K+1}^{(1)},
$$

where

$$
\begin{aligned}
& \mathbf{M}^{(K)}= {\left[\begin{array}{l}
\mathbf{M}^{(K-1)} \\
\mathbf{M}^{(K-1)}
\end{array}\right] \quad \text { with } \quad \mathbf{M}^{(1)}=\mathbf{I}_{2 L} } \\
& \mathbf{A}^{(K)}= {\left[\begin{array}{lr}
\mathbf{A}^{(K-1)} & \mathbf{0} \\
\mathbf{0} & \mathbf{A}^{(K-1)}
\end{array}\right] \quad \text { with } } \\
& \mathbf{A}^{(0)}=\text { blockdiag }\left\{\left[\begin{array}{l}
1 \\
1
\end{array}\right]\right\} \in \mathbb{Z}^{2^{L} \times 2^{L-1}} \\
& \mathbf{D}^{(K)}=\left[\begin{array}{lr}
\mathbf{D}^{(K-1)} & \mathbf{0} \\
\mathbf{0} & \mathbf{D}^{(K-1)}
\end{array}\right] \text { with } \mathbf{D}^{(1)}=\operatorname{diag}\{\mathbf{w}\} .
\end{aligned}
$$

This form of the matched filter bank receiver is depicted in the flow graph in Fig. 1. To determine the correct output bit, the arg max operator in (4) would operate on $\mathbf{y}_{k}^{(K)}$.

\subsection{Computational Complexity}

Inspecting the operations in Fig. 1, $2^{L}$ matched filter operations of length $N$ have to be performed per bit period. As the matrices $\mathbf{M}^{(k)}$ and $\mathbf{A}^{(k)}$ only perform indexing, the only arithmetic operations required are multiplications with the diagonal elements of the phase correction matrices $\mathbf{D}^{(K)}$, yielding a total of

$$
C_{\text {efficient }}=2^{L+2} N+4 \sum_{k=1}^{K-1} 2^{L+k} \lesssim 2^{L+2} N+2^{L+K+2}
$$

MACs. If marginal bits are disregarded analogously to the matched filter receivers in $[1,8]$ as discussed in Sec. 3, then desired outputs $\tilde{\mathbf{y}}_{k}^{(K)}=\mathbf{S}^{(K)} \mathbf{y}_{k}^{(K)}$ can be extracted. As an example for $L=3$, the extraction matrix $\mathbf{S}^{(K)}$ takes the form

$$
\begin{aligned}
\mathbf{S}^{(K)} & =\left[\begin{array}{ll}
\mathbf{G}^{(K)} & \mathbf{0}_{2^{K} \times 2^{K}+1}
\end{array}\right] \quad \text { with } \\
\mathbf{G}^{(K)} & =\operatorname{blockdiag}\left\{\left[\begin{array}{ll}
1 & 0
\end{array}\right]\right\} \in \mathbb{Z}^{2^{K} \times 2^{K+1}} .
\end{aligned}
$$

The extraction matrices can be appropriately absorbed into (19)(21), yielding a reduced complexity of

$$
\tilde{C}_{\text {efficient }} \lesssim 2^{L+2} N+2^{K+3} \quad[\text { MACs }]
$$

\section{CARRIER FREQUENCY OFFSET CORRECTION}

An estimation of the carrier frequency offset can be based on the received signal in (3) by denoting

$$
\begin{aligned}
\mathcal{E}\left\{r[n] r^{*}[n-M]\right\}= & |A|^{2} \mathcal{E}\left\{s[n] s^{*}[n-M]\right\} e^{j \Delta \Omega M}+ \\
& +\mathcal{E}\left\{v[n] v^{*}[n-M]\right\} \\
= & |A|^{2} e^{j \Delta \Omega M}
\end{aligned}
$$

where $\mathcal{E}\{\cdot\}$ is the expectation operator. By selecting $M$ sufficiently large, the autocorrelation term of the noise in (25) vanishes. Since the instantaneous angular frequency accumulated over $M$ samples of the transmitted signal $s[n]$ will either rotate in a positive or negative direction but on average be zero, we have $\mathcal{E}\left\{s[n] s^{*}[n-M]\right\}=1$. Note that the detection of the carrier frequency offset is independent of any other receiver functions.

\subsection{Cost Function}

We create a modified receiver input $\tilde{r}[n]$,

$$
\tilde{r}[n]=\operatorname{Br}[n] e^{j \Theta n},
$$

i.e. modulating by $\Theta$ and scaling the input by $B$, which is ideally selected such that $B^{-1}=|r[n]|=A$, and $\Theta$ to match the carrier offset $\Delta \Omega$. In order to determine $\Theta$, we can use the following constant modulus $(\mathrm{CM})$ cost function,

$$
\chi=\left|\mathcal{E}\left\{\tilde{r}[n] \tilde{r}^{*}[n-M]\right\}-1\right|^{2} .
$$

Inserting (27) and (26) with $B^{-1}=|r[n]|$ into (28) yields

$$
\begin{aligned}
\chi= & 1+|A B|^{4}-2|A B|^{2} \cos ((\Theta+\Delta \Omega) M) \\
\text { with } \chi=0 & (29)
\end{aligned}
$$

We are interested in the solution for $k=0$ only, for which the cost function provides a unique minimum under the condition

$$
-\pi<(\Theta+\Delta \Omega) M<\pi
$$

similar to [7]. Hence, a trade-off exists for the selection of $L$ between decorrelating the noise in the receiver and not exceeding the bounds in (30).

\subsection{Stochastic Gradient Method}

Within the bounds of (30), $\Theta$ can be iteratively adapted over time based on gradient descend techniques according to

$$
\Theta[n+1]=\Theta[n]-\mu_{\Theta} \frac{\partial \chi}{\partial \Theta}
$$

with a suitable step size parameter $\mu_{\Theta}$. A stochastic gradient can be based on an instantaneous cost $\hat{\chi}$ by omitting expectations in (28) and assuming small changes in $\Theta$ only:

$$
\frac{\partial \hat{\chi}}{\partial \Theta}=-2 M \Im\left\{\tilde{r}[n] \tilde{r}^{*}[n-M]\left(\tilde{r}[n] \tilde{r}^{*}[n-M]-1\right)^{*}\right\} .
$$

Fig. 1. Low-complexity implementation of a matched filter bank high-performance GFSK receiver. The received GFSK signal $r[n]$ is passed through a serial/parallel converter and a filter bank $\mathbf{W}^{(1)}$ with a single bit duration. Processed over $K$ stages, the matched filter bank outputs are contained in $\mathbf{y}_{k}^{(K)}$.

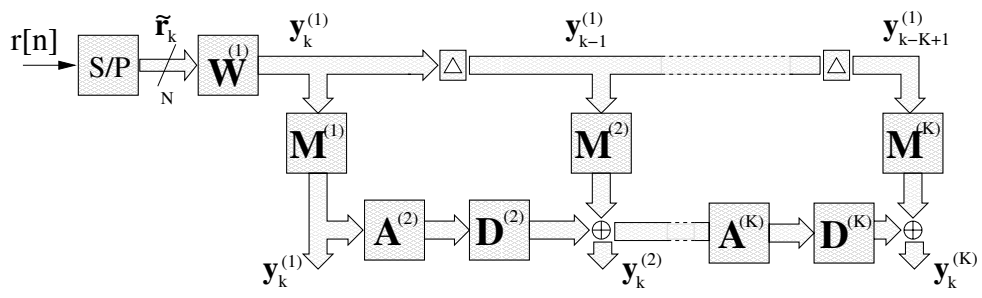




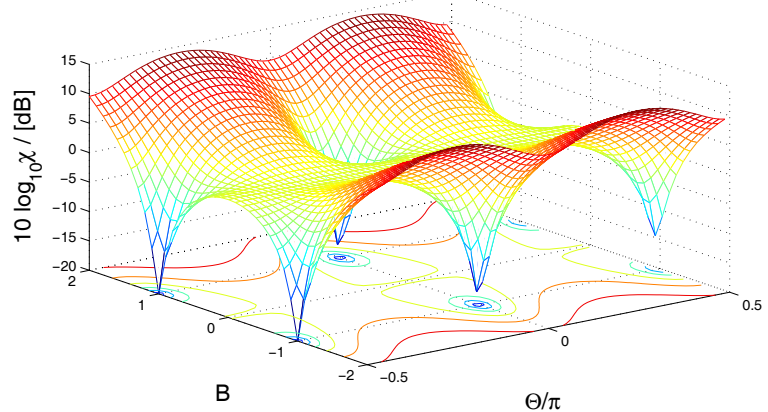

Fig. 2. Cost function $\chi$ for $A=1, \Delta \Omega=0$, and $M=4$.

\begin{tabular}{|l||c|c|c|c|}
\hline$K$ & 3 & 5 & 7 & 9 \\
\hline \hline$C_{\text {standard }} /[\mathrm{MAC}]$ & 192 & 1280 & 7168 & 36864 \\
\hline$\tilde{C}_{\text {efficient }} /[\mathrm{MAC}]$ & 96 & 288 & 1056 & 4128 \\
\hline
\end{tabular}

Table 1. Bluetooth receiver complexity with $L=3$ and $N=2$.

Similarly, the gain parameter $B$ in (27), can be estimated by

$$
B[n+1]=B[n]-\mu_{B} \frac{\partial \hat{\chi}}{\partial B},
$$

whereby the stochastic gradient analogously to above results in

$$
\frac{\partial \hat{\chi}}{\partial B}=\frac{4}{B} \Re\left\{\tilde{r}[n] \tilde{r}^{*}[n-M]\left(\tilde{r}[n] \tilde{r}^{*}[n-M]-1\right)^{*}\right\} .
$$

The modified received signal $\tilde{r}[n]$ in (27) would then be passed into the matched filter detector discussed in Sec. 4 instead of $r[n]$.

\section{RESULTS}

We show some results exemplarily for Bluetooth, which requires for its specified bandwidth-time product of 0.5 a Gaussian filter with support $L=3$. Further, we have chosen $N=2$ throughout.

\subsection{Matched Filter Performance}

The receiver improves with the increase in the observation interval length $K$ [1]. Bluetooth demands a maximum BER of $10^{-3}$, which relatively simple algorithms achieve at $14.8 \mathrm{~dB}$ channel SNR [9], while some practitioners even assume $21 \mathrm{~dB}$ to be required [10]. As shown in Fig. 3, the matched filter receiver with $K=9$ can operate in an SNR of $9.8 \mathrm{~dB}$, highlighting the improved performance.

The computational cost for standard and efficiently implemented matched filter receivers is compared in Tab. 1, with the proposed method only requiring about $11 \%$ of the standard method in [1].

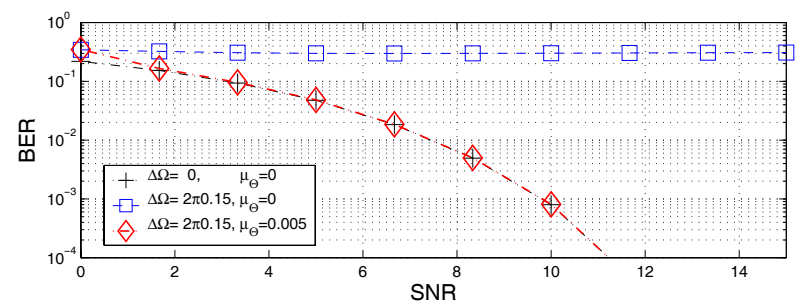

Fig. 3. Effect of carrier offset correction on BER for GFSK with bandwidth-time product $0.5, h=0.35, K=9, N=2$ and $M=2$.

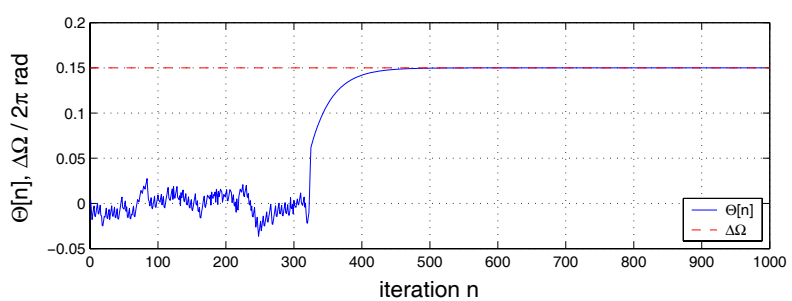

Fig. 4. Learning curve of carrier offset correction according to (31) for GFSK $K=9, M=2, \mu_{\Theta}=0.005$ and $\Delta \Omega=2 \pi 0.15$.

\subsection{Carrier Frequency Offset}

Bluetooth permits a carrier offset of up to $75 \mathrm{kHz}$, which can severely degrade performance [4], and for $N=2$ translates into a maximum normalised carrier offset $|\Delta \Omega|=2 \pi 0.15$. The matched filter receiver, while performing near-optimum in AWGN, suffers under carrier offset conditions, which is shown in Fig. 3 by the BER curve with carrier offset but no correction. Applying the algorithm derived in Sec. 5 allows to adapt to the correct carrier offset, with the learning curve given in Fig. 4, resulting in a near optimum BER performance of the matched filter according to Fig. 3.

\section{CONCLUSION}

We have considered high performance matched filter detectors for GFSK modulated signals. By analysing the possible transmitted sequences, a recursive low-cost implementation has been found. For popular transmission schemes such as Bluetooth, where expensive receiver algorithms are prohibitive, the proposed receiver can operate with identical performance but at a considerably reduced computational cost.

Frequency errors seriously degrade performance of the highperformance receiver. We have proposed a blind adaptation scheme to correct for carrier frequency offset, which are fast converging and permit near optimum receiver performance in AWGN.

\section{REFERENCES}

[1] W.P. Osborne and M.B. Luntz, "Coherent and Noncoherent Detection of CPFSK," IEEE Trans. Comms., COM-22(8):1023-1036, 1974.

[2] T. Aulin, N. Rydbeck, and C.-E.W. Sundberg, "Continuous Phase Modulation-Part II: Partial Response Signaling," IEEE Trans. Comms, COM-29(3):210-225, 1981.

[3] B.A. Carlson, Communication Systems, McGraw-Hill, 3rd ed., 1986.

[4] C. Robinson and A. Purvis, "Demodulation of Bluetooth GFSK Signals Under Carrier Frequency Error Conditions," Proc. IEE Colloq. DSP Enabled Radio, Livingston, UK, Sept. 2003.

[5] A.N.D. Andrea, A. Ginesi, and U. Mengali, "Frequency Detector For CPM," IEEE Trans. Comms, COM-43(2):1828-1837, 1995.

[6] P. Spasojevic and C.N. Georghiades, "Blind Frequency Compensation For Binary CPM with $\mathrm{h}=1 / 2$ and A Positive Frequency Pulse," Proc. Globecom, Sidney, Nov. 1998.

[7] A. Aziz, P. Spasojevic, and C.N. Georghiades, " Large Frequency Offset Compensation for GMSK Signals: DSP Receiver Implementation," Proc. Int. Conf. Sig. Proc. Applications, Toronto, June 1998.

[8] T.A. Schonhoff, "Symbol Error Probabilities for M-ary CPFSK: Coherent and Noncoherent Detection," IEEE Trans. Comms, COM24(6):644-652, 1976.

[9] R. Schiphorst, F. Hoeksema, and K. Slump, "Bluetooth Demodulation Algorithms and their Performance," Proc. Workshop Software Radios, Karlsruhe, pp. 99-105, 2002.

[10] Ericsson, Ericsson Bluetooth Development Kit documentation, October 1999 